\title{
Predicting future handedness and hemispheric dominance during infancy by analyzing sleep spindles
}

\author{
Ali H. Alwadei, MD, Turki F. AlOsaimi, MD, Manar A. Bayounis, MD, Ghadd A AlHajaj, MD, Fahad A. Bashiri, MD.
}

\begin{abstract}
الأهداف : إكتشاف تواؤم مغزل النوم باستخدام تخطيط المخ لدى

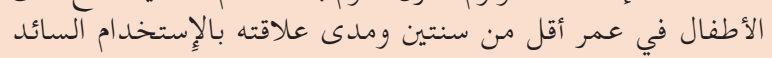
لإِحدى اليدّين مستقبلا.

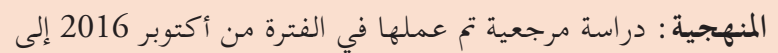

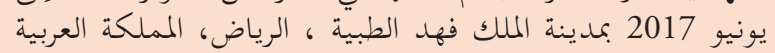

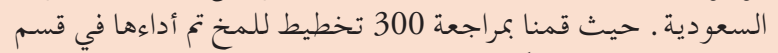

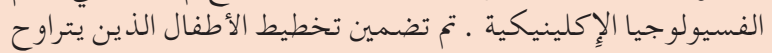

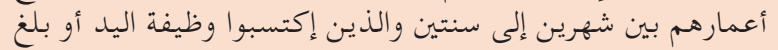

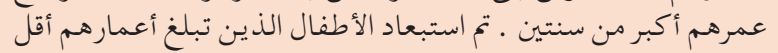

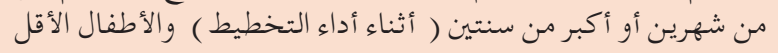

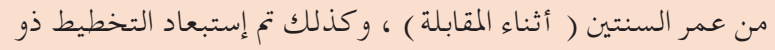

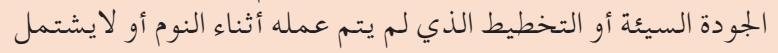

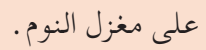

النتائج : وجدانا بأن تواؤم مغزل النوم كان سائدا في الفص الأيمكن

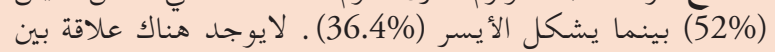

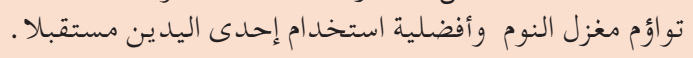

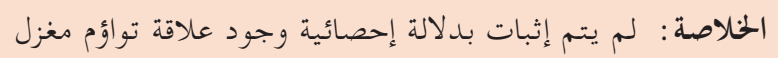

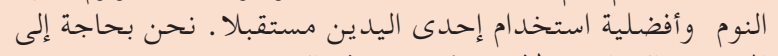
المزيد من الدراسات لإِثبات أو نفي هذه الفيد الفرضية.

Objectives: To investigate whether sleep spindles asynchrony (SSA) using scalp sleep electroencephalogram (EEG) among children below 2 years of age can predict future handedness.

Methods: This is a retrospective study conducted from October 2016 until June 2017 at the King Fahad Medical City (KFMC), Riyadh, Kingdom of Saudi Arabia. We retrospectively reviewed 300 EEGs recorded at our neurophysiology laboratory. We included EEGs performed during sleep for infants aged 2 months to 2 years who have already attained their handedness or those aged above 2 years. We excluded records of children younger than 2 months or above 2 years of age (at the time of the
\end{abstract}

EEG) or those aged below 2 years (at the time of the interview), and severely abnormal tracings and those without sleep or enough SSA .

Results: The lateralization of Sleep Spindles (SS) was mostly right-hemispheric (52\%) compared to lefthemispheric (36.4\%). The overall SS laterality did not correlate with handedness $(p=0.81)$. In the majority of right-handed (64\%) and left-handed (60\%) children, the SSA was contralateral to the side of hand preference; however, it did not correlate statistically $(p=0.377)$.

Conclusion: We were unable to prove a statistically significant correlation between SSA and future hand preference. Further research involving larger cohorts is still needed.

Neurosciences 2020; Vol. 25 (3): 188-192 doi: 10.17712/nsj.2020.3.20190129

From the Pediatric Neurology Department (Alwadei, AlOsaimi), National Neuroscience Institute, King Fahad Medical City, Department of Pediatrics (Bayounis), Al-Yamama Hospital, Division of Pediatric Neurology (Bashiri), Department of Pediatrics, College of Medicine, King Khalid University Hospital, King Saud University and from the Department of Pediatrics (AlHajaj), Unaizah College of Medicine and Medical Sciences, Qassim University, Unaizah, Qassim, Kingdom of Saudi Arabia

Received 29th December 2019. Accepted 29th February 2020.

Address correspondence and reprint request to: Dr.Fahad A. Bashiri, Division of Pediatric Neurology, Department of Pediatrics, College of Medicine, King Saud University, Riyadh, Kingdom of Saudi Arabia. E-mail:fbashiri@ksu.edu.sa

ORCID ID: https://orcid.org/0000-0003-1436-6727

Sleep architecture is an essential part of evaluating $S_{\text {any }}$ electroencephalogram (EEG). Despite being recognized and described as early as the discovery of EEG, sleep features remain understudied. In most children, Sleep Spindles (SS) do not synchronize over the 2 hemispheres until the child develops Handedness, 
usually at 2 years of age. This coincidence of the establishment of Handedness and synchrony of SS makes the connection between the 2 maturational developments important. We planned to investigate the possibility of anticipating future hemispheric dominance and future Handedness using the concept of Sleep Spindles Asynchrony (SSA). The SS consist of bilaterally symmetric low to medium voltage bicentral synchronous rhythmic spindle-like waves, with frequency ranging from 12 to $16 \mathrm{~Hz}$, which often appear along K-complexes lasting approximately 2-3 seconds. ${ }^{1}$ The identification of SS is easy in any sleep EEG due to their abundance in infants and children. Their typical location in adults is a synchronous burst of waves around the vertex; in children, it varies according to age. ${ }^{2-7}$ It is thought that the origin of sleep spindles is the thalamocortical network. However, there is no functional meaning definitively established for this mechanism so far, which makes our study relevant to this area. ${ }^{3}$ The SS are not seen in the first 2 months of life and start to appear afterward. ${ }^{8}$ The absence of sleep spindles during mid-infancy is considered a significant abnormality. However, their independent occurrence (asynchrony) below 2 years of age is common and a normal finding. ${ }^{9-12}$ These sleep milestones are consistent between subjects, and therefore they are an essential indication of functional maturation. ${ }^{13}$ Chatburn et a ${ }^{14}$ found that sleep spindle activity was associated with various aspects of maturation and is an integral part of brain development. It is suggested that sleep EEG may be a marker for intellectual ability. ${ }^{15}$ Specifically, SS are associated with the intelligence quotient (IQ). ${ }^{16}$ The SS are also found to be involved in procedural memory. ${ }^{17}$ There are a few speculations about how Handedness is created in individuals. Although antenatal mechanisms might be essential, analysis considering in utero development verified that Handedness was an extremely accurate indicator of hemispheric dominance after birth. ${ }^{18}$ Early hand preference does not develop before one-year of age, and the usual age at which Handedness appears is 2 years, coinciding electrographically with SS synchrony, making a causal relationship or mechanism between the 2 markers highly likely. ${ }^{19-20}$ There is no previously published research to date to have studied this area. ${ }^{21}$

Disclosure. Authors have no conflict of interests, and the work was not supported or funded by any drug company.
Methods. This study was retrospectively conducted from October 2016 until June 2017 at King Fahad Medical City (KFMC), National Neuroscience Institute (NNI), Riyadh, Kingdom of Saudi Arabia. The study involved children aged between 2 months and 2 years who did an EEG during 2014 and 2015 that are approved to be reported independently by 2 qualified neurophysiologists.

The inclusion criteria (IC) were: children (males and females) who underwent sleep EEG at or after 2 months to 2 years of age, currently aged above 2 years to ensure Handedness. The EEG inclusion criteria were: EEG records were carried out in a neurophysiology laboratory for 20 minutes or more with achieved sleep tracing according to the standardized electrode placement system, which is the international 10-20 placement system with at least 2 montages (one bipolar montage and one unipolar/referential montage). The SS observed are those localized on frontocentral and centroparietal leads with a frequency of $12-16 \mathrm{~Hz}$ lasting for 1-5 seconds. Asynchronous SS were defined as spindles that appeared independently on either hemisphere with a time lag of at least half a second. The exclusion criteria were: patients underwent an EEG study before 2 months or after 2 years of age, current age of the patient was less than 2 years, and patients were deceased at the time of the interview. The EEG

Table 1 - Patient characteristics.

\begin{tabular}{lc}
\hline Characteristics & $\mathrm{n}(\%)$ \\
\hline Age at EEG time (yr.) & $20(60.6)$ \\
$\leq 1$ & $13(39.4)$ \\
$1-2$ & \\
Present age (yr.) & $15(45.5)$ \\
$<3$ & $18(54.5)$ \\
$3-4$ & \\
Male & $14(42)$ \\
14 & $19(58)$ \\
Female & $12(36.4)$ \\
19 & $17(51.5)$ \\
Asynchronous sleep spindles before the age of 2 years \\
Left & $4(12.1)$ \\
Right & \\
Inconclusive & $5(15.2)$ \\
Handedness & $11(33.3)$ \\
Left & $17(51.5)$ \\
Right & EEG - Electroencephalogram \\
Inconclusive & \\
\hline \multicolumn{2}{r}{}
\end{tabular}


Table 2 - EEG laterality observed across handedness.

\begin{tabular}{|c|c|c|c|c|c|}
\hline \multirow[t]{2}{*}{ Handedness } & \multicolumn{3}{|c|}{$\begin{array}{c}\text { Asynchronous sleep spindles before the } \\
\text { age of } 2 \text { years }\end{array}$} & \multirow[t]{2}{*}{ Total } & \multirow[t]{2}{*}{$p$-value } \\
\hline & Left & Right & Inconclusive & & \\
\hline Left & $2(16.7)$ & $3(17.6)$ & $0(0)$ & $5(15.2)$ & 0.377 \\
\hline Right & $5(41.7)$ & $6(35.3)$ & $1(25.0)$ & $12(36.4)$ & \\
\hline Inconclusive & $5(41.7)$ & $9(52.9)$ & $3(75.0)$ & $17(51.5)$ & \\
\hline Total & $12(36.4)$ & $17(51.5)$ & $4(12.1)$ & $33(100)$ & \\
\hline \multicolumn{6}{|c|}{ Handedness observed across EEG laterality } \\
\hline Left & $2(50.0)$ & $2(50.0)$ & $0(0)$ & $4(12.1)$ & 0.377 \\
\hline Right & $5(41.7)$ & $6(50.0)$ & $1(8.3)$ & $12(36.4)$ & \\
\hline Inconclusive & $5(29.4)$ & $9(52.9)$ & $3(17.6)$ & $17(51.5)$ & \\
\hline Total & $12(36.4)$ & $17(51.5)$ & $4(12.1)$ & $33(100)$ & \\
\hline
\end{tabular}

exclusion criteria were: grossly abnormal EEGs with abundant epileptiform discharges and electrographic seizures, EEG records acquired without sleep tracing, and in patients aged less than 2 months or more than 2 years. We also excluded EEGs with solely synchronous SS, spindles that do not fulfill the criteria of described SS or very few SSA defined by cut off of less than $10 \%$ difference. We then reviewed a total of 300 EEGs in 3 stages: 1) Analysis of the whole tracing, excluding the grossly abnormal EEGs. 2) Exclusion of records that did not meet our IC. During the first two stages, 267 EEGs were excluded. 3) Carefully analyzing the sleep spindles in the remaining 33 recordings by quantifying a total of 1115 recorded spindles, including left-sided asynchronous, right-sided asynchronous, and bilaterally synchronous SS.

The KFMC ethics committee approved the study design and proposal. Informed consent was obtained during the voluntary participation in the study by text message followed by a phone call to confirm the parents' willingness to participate in the research study. Parents were later contacted again by phone to confirm Handedness. They were also asked about current age and developmental milestones, family, and personal history of Handedness by asking the parents about the daily behaviors of the child, such as eating, drawing, and coloring. In the end, 33 patients met our IC, and their data were tabulated in Excel sheets, and statistical analysis was performed using SPSS 17 (SPSS, Inc., Chicago, Illinois, United States). Categorical data were described as frequency (percent), and the metric data was measured as the mean (standard deviation). The association between sleep spindles and all the independent factors was measured by chi-squared test
Table 3 - Asynchrony observed across handedness.

\begin{tabular}{lccc}
\hline Using Chi-square & Right handed & Left handed & Total \\
\hline Left-sided sleep spindle & 7 & 2 & 9 \\
Right-sided sleep spindle & 4 & 3 & 7 \\
Total & 11 & 5 & 16 \\
& & P-Value & \\
Pearson's Chi-square: & 0.780 & 0.377 & \\
With Yate's continuity & & & \\
correction: & 0.115 & 0.734 & \\
\hline
\end{tabular}

$\left(\chi^{2}\right)$ and Kendall's tau b correlation. All the inferences were drawn at a $95 \%$ confidence interval, and Statistical significance was defined as $p<0.05$.

Results. After reviewing 300 EEG records, 33 EEGs during sleep and wakefulness met our IC in which we analyzed 1115 bursts of synchronous and asynchronous sleep spindles. Of these, $344(31 \%)$ started over the right hemisphere, $411(36.8 \%)$ started over the left hemisphere, and $360(32.3 \%)$ were synchronous. A total of 33 patients met our IC (19;57.6\% females and 14; $42.4 \%$ males). Patient characteristics are summarized in Table 1.

Our findings show that, of 33 patients, 5 (15.2\%) were left-handed, $12(36.4 \%)$ were right-handed, and $16(48.5 \%)$ patients had no preferred handedness. The EEG results showed that: $12(36.4 \%)$ patients had left-sided sleep spindles, 17 (51.5\%) patients had right-sided sleep spindles, and $4(12.1 \%)$ patients had inconclusive sleep spindles (Table 1). As for EEG laterality (Table 2), we observed that: A) of 12 patients with predominantly left-sided SS, $5(41.7 \%)$ attained right-handedness, 2 (16.6\%) attained left-handedness, 


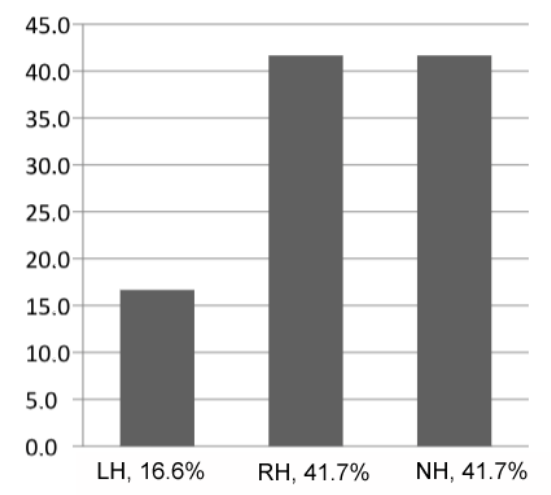

Figure 1 - Handedness among those with predominantly left-sided sleep spindles. RH - right handed, LH - left handed, NH Not identified Handedness.

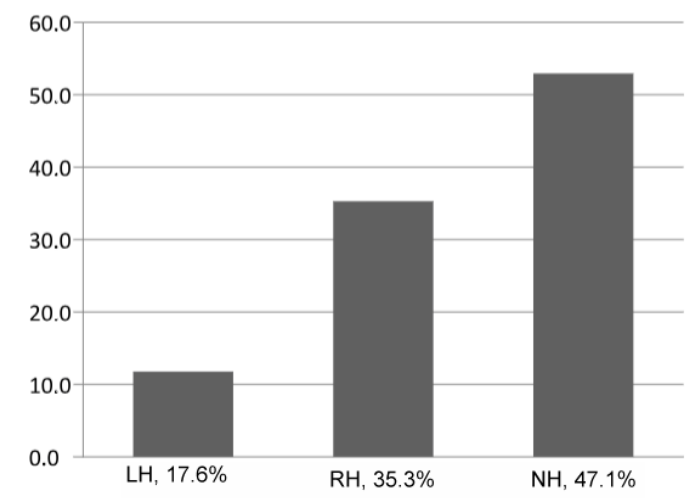

Figure 2 - Handedness among those with predominantly right-sided sleep spindles. RH - Right Handed, LH - left handed, NH Not identified Handedness.

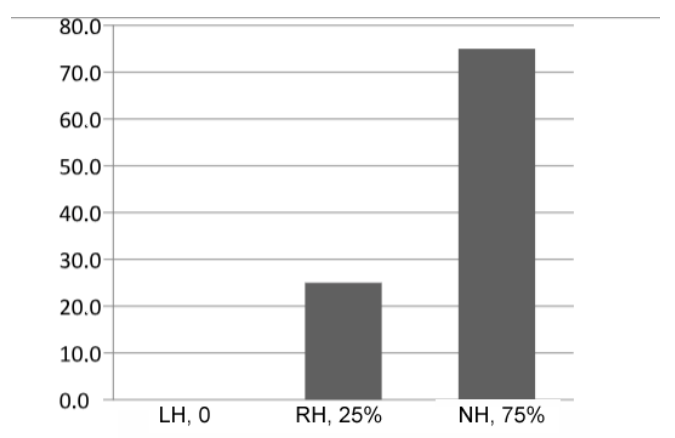

Figure 3 - Handedness among those with predominantly inconclusive spindles. RH- Right Handed, LH - Left Handed, NH - Not identified Handedness

and $5(41.7 \%)$ did not attain Handedness (Figure 1). B) of 17 patients with predominantly right-sided SS, $6(35.3 \%)$ attained right-handedness, 3 (17.6\%) attained left-handedness, and $8(47.1 \%)$ did not attain
Handedness (Figure 2). C) of the remaining 4 patients with inconclusive sleep spindles, $1(25 \%)$ attained right-handedness, none (0\%) attained left-handedness, and $3(75 \%)$ had undetermined Handedness (Figure 3). We investigated the Handedness observed across EEG laterality ( $p=0.377)$ (Table 2). Out of 33 patients: A) of 5 left-handed patients, 2 (40\%) had left-sided SS and $3(60 \%)$ had right-sided SS; B) of 12 right-handed patients, $5(41.7 \%)$ had left-sided SS, $6(50 \%)$ had right-sided SS, and $1(8.3 \%)$ had inconclusive SS; C) of 16 patients who did not develop handedness, 5 (31.3\%) had left-sided SS, 8 (50\%) had right-sided SS, and the last 3 had inconclusive SS. Excluding the 4 inconclusive patients, further dichotomous analysis of the 29 patients with right-sided or left-sided spindles revealed that, among the 11 right-handed children, the majority $(7 / 11 ; 64 \%)$ had SS predominantly leading from the contralateral left hemisphere, and among the five left-handed children, the majority $(3 / 5 ; 60 \%)$ also had SS predominantly leading from the contralateral right hemisphere. Finally, among the remaining 13 children who were not handed, the majority $(7 / 13 ; 53.8 \%)$ of the spindles were synchronous (Table 3 ).

Discussion. In this study, we hypothesized that SSA might anticipate Handedness during the first 2 years of life as a new readily available clinical tool, considering the daily medical practice and current literature. The purpose of this paper was to find an easy and noninvasive predictor or a biomarker of hemispheric dominance. Handedness was chosen as a clinical sign of cerebral lateralization to help in future decisionmaking during the evaluation of young children undergoing epilepsy surgical intervention as a pivotal practical implication. Some studies addressed question on spindles in adults whose synchronization of SS has already been achieved. ${ }^{22}$ Analyzing these results showed that more than half of the left-handed patients had right-sided sleep spindles, and most of the right-handed patients previously had left-sided SS. More than half of our sample had not developed Handedness, which could be attributed to most of the included children being referred to tertiary care centers for developmental delay, seizures, or mental/neurological disability. Our results did not show a statistically significant correlation between SSA and Handedness, thus cannot be a reliable predictor of hemispheric dominance. Other studies found a relationship among gender, age, type of sleep spindle, and their distribution over the cerebral hemisphere, showing that young females are characterized by left-hemispheric dominance in fast sleep spindling and older males are characterized by more 
pronounced left temporal/orbitofrontal dominance of fast sleep spindling. ${ }^{22}$ Our study was limited by a few factors, including the small sample size and the fact that patients were mostly neurologically impaired. Some patients were not included in the data analysis due to difficulty reaching the caregiver, death of the patient, or poor EEG recording. All our patients were referred for a tertiary care evaluation; thus, the majority $(25 / 33 ; 76 \%)$ was developmentally impaired. While conducting this study, only the side of SS onset was taken into consideration for asynchrony assessment; we have not used other measurement parameters, including density, frequency, and amplitude. Although a $p$-value of 0.377 suggests no statistically significant correlation, we hope that our study inspires further work in this area, and we believe that if our sample size was 100 or more children with normal development, it might have shown a more significant conclusion. Future research directions include recruiting many children with normal development who are referred for sleep EEG for either benign seizures or seizure mimickers. Also, additional waveform features other than timing (synchrony), such as amplitude, frequency, and duration should be added to future work.

In conclusion, although a potential significance has been shown by this paper, analyzing sleep spindle asynchrony (SSA) did not yield statistically significant values to predict future Handedness. Further research on larger cohorts is still needed to determine the final implications of clinical practice.

Acknowledgment. The authors would like to thank Mr. Wani TA for statistical analysis. We want to thank Editage for English language editing.

\section{References}

1. Pavlidis E, Lloyd RO, Mathieson S, Boylan GB. A review of important EEG features for the assessment of brain maturation in premature infants. Acta Paediatr 2017; 106: 1394-1408.

2. Rechtschaffen A, Kales A. A Manual of standardized terminology, techniques and scoring system for sleep stages of human subjects. Washington (DC): US Government Printing Office; 1968.

3. De Gennaro L, Ferrara M. Sleep spindles: an overview. Sleep Med Rev 2003; 7: 423-440.
4. Libenson M, editor. Practical approach to electroencephalography. Philadelphia (PA): Saunders; 2010.

5. Jobert M, Poiseau E, Jahnig P, SchulzH, Kubicki S. Topographical analysis of sleep spindle activity. Neuropsychobiology 1992; 26: 210-217.

6. Werth E, Achermann P, Borbely A. Fronto-occipital EEG power gradients in human sleep. J Sleep Res 1997; 6: 102-112.

7. Zeitlhofer J, Gruber G, Anderer P, Asenbaum S, Schimicek P, Saletu B. Topographic distribution of sleep spindles in young healthy subjects. J Sleep Res 1997; 6: 149-155.

8. Metcalf DR. EEG sleep spindle ontogenesis. Neuropadiatrie 1970; 1: 428-433.

9. Louis J, Zhang JX, Revol M, Debilly G, Challamel MJ. Ontogenesis of nocturnal organization of sleep spindles: a longitudinal study during the first 6 months of life. Electroencephalogr Clin Neurophysiol 1992; 83: 289-296.

10. Louis J, Cannard C, Bastuji H, Challamel MJ. Sleep ontogenesis revisited: a longitudinal 24-hour home polygraphic study on 15 normal infants during the first two years of life. Sleep 1997; 20: 323-333.

11. Hagne I. Development of the Sleep EEG in Normal Infants During the First Year of Life. Acta Paediatr 1972; 61: 25-53.

12. Wu HS, Gould JB, Lee AF, Fineberg N. Factors affecting sleep spindle activity during infancy. Dev Med Child Neurol 1980; 22: 344-351.

13. Greenfield L, Geyer J, Carney P, editors. Reading EEGs: A Practical Approach. Lippincott Williams \& Wilkins; 2012.

14. Chatburn A, Coussens S, Lushington K, Kennedy D, Baumert M, Kohler M. Sleep spindle activity and cognitive performance in healthy children. Sleep 2013; 36: 237-243.

15. Geiger A, Huber R, Kurth S, Ringli M, Jenni OG, Achermann P. The Sleep EEG as a Marker of Intellectual Ability in School Age Children. Sleep 2011; 34: 181-189.

16. Fogel SM, Nader R, Cote KA, Smith CT. Sleep spindles and learning potential. Behav Neurosci 2007; 121: 1-10.

17. Fogel SM, Smith CT. Learning-dependent changes in sleep spindles and Stage 2 sleep. J Sleep Res 2006; 15: 250-255.

18. Michel GF, Goodwin R. Intrauterine birth position predicts newborn supine head position preferences. Infant Behavior \& Development 1979; 2: 29-38.

19. Geschwind N, Galaburda AM. Cerebral lateralization. Biological mechanisms, associations, and pathology: I. A hypothesis and a program for research. Arch Neurol 1985; 42: 428-459.

20. Knecht S, Drager B, Deppe M, Bobe L, Lohmann H, Flöel A, et al. Handedness and hemispheric language dominance in healthy humans. Brain 2000; 123: 2512-2518.

21. Payne MA. Impact of cultural pressures on self-reports of actual and approved hand use. Neuropsychologia 1987; 25: 247-258.

22. Bodizs R, Gombos F, Ujma PP, Szakadat S, Sandor P, Simor $\mathrm{P}$, et al. The hemispheric lateralization of sleep spindles in humans. Sleep Spindles \& Cortical Up States 2017; 1: 42-54. 